\title{
Impact of growth temperature on InAs/GalnSb strained layer superlattices for very long wavelength infrared detection
}

\author{
H. J. Haugan, ${ }^{1, \text { a) }}$ G. J. Brown, ${ }^{1}$ S. Elhamri, ${ }^{2}$ W. C. Mitchel, ${ }^{1}$ K. Mahalingam, ${ }^{1}$ M. Kim, ${ }^{1}$ \\ G. T. Noe, ${ }^{3}$ N. E. Ogden, ${ }^{3}$ and J. Kono ${ }^{3}$ \\ ${ }^{1}$ Air Force Research Laboratory, Materials \& Manufacturing Directorate, Wright-Patterson Air Force Base, \\ Ohio 45433, USA \\ ${ }^{2}$ Department of Physics, University of Dayton, Ohio 45469, USA \\ ${ }^{3}$ Department of Electrical and Computer Engineering, Rice University, Houston, Texas 77005, USA
}

(Received 14 September 2012; accepted 11 October 2012; published online 23 October 2012)

\begin{abstract}
We explore the optimum growth space for a $47.0 \AA \mathrm{InAs} / 21.5 \AA \mathrm{Ga}_{0.75} \mathrm{In}_{0.25} \mathrm{Sb}$ superlattices (SLs) designed for the maximum Auger suppression for a very long wavelength infrared gap. Our growth process produces a consistent gap of $50 \pm 5 \mathrm{meV}$. However, SL quality is sensitive to the growth temperature $\left(\mathrm{T}_{\mathrm{g}}\right)$. For the SLs grown at $390-470{ }^{\circ} \mathrm{C}$, a photoresponse signal gradually increases as $\mathrm{T}_{\mathrm{g}}$ increases from 400 to $440^{\circ} \mathrm{C}$. Outside this temperature window, the SL quality deteriorates very rapidly. All SLs were n-type with mobility of $\sim 10000 \mathrm{~V} / \mathrm{cm}^{2}$ and $300 \mathrm{~K}$ recombination lifetime of $\sim 70 \mathrm{~ns}$ for an optimized SL. (C) 2012 American Institute of Physics.

[http://dx.doi.org/10.1063/1.4764015]
\end{abstract}

An alternative infrared material system proposed by Smith and Mailhiot ${ }^{1}$ uses the concept of broken-gap type-II band alignment of the InAs/GaInSb strained layer superlattices (SLs) to achieve narrow gaps for the very long wavelength infrared (VLWIR) detection $(>14 \mu \mathrm{m})$. By alloying indium to the GaSb layer, the lattice constant of the GaInSb layer increases. The biaxial tension in the InAs layer lowers the conduction band, while the biaxial compression in the GaInAs layer raises the heavy-hole (HH) band. As a result, the very narrow gap can be achieved without sacrificing optical absorption. In addition, an intentionally introduced strain can create a large splitting between the $\mathrm{HH}$ and light-hole bands in the p-type SLs; this situation can prevent the holehole Auger recombination process, therefore the Auger limited minority carrier lifetime, detectivity, and the background limited operating temperature can be significantly improved. Grein et al. ${ }^{2}$ demonstrated how a small variation in the strain splitting between the uppermost valence bands can create an order of magnitude differences on their calculated detectivities. For the SLs designed for the same $80 \mathrm{meV}$ gap at $40 \mathrm{~K}$, the electronic band structure of either $49.7 \AA$ InAs/57.0 $\mathrm{Ga}_{0.9} \mathrm{In}_{0.1} \mathrm{Sb}$ or $47.0 \AA$ InAs/21. $5 \AA \mathrm{Ga}_{0.75} \operatorname{In}_{0.25} \mathrm{Sb}$ SL designs computed with the interface terms had the total lifetime of either $5 \times 10^{-9}$ or $1.4 \times 10^{-7} \mathrm{~s}$. This difference leads the device detectivity to be either $5.2 \times 10^{13}$ or $6.0 \times 10^{14} \mathrm{cmHz}^{1 / 2} / \mathrm{W}$. Although the greater strain splitting generates the better detectivity, one has to limit the indium alloy composition below $30 \%$ due to difficulties in strain balancing above this percentage. The authors also computed device detectivity for the more realistic case of $82 \mathrm{meV}$ SL detectors with a $35 \mathrm{~ns}$ Shockley-Read-Hall (SRH) lifetime. ${ }^{3}$ Unexpectedly, Auger processes were fast enough to overrule SRH processes and the detectors still can operate at reasonably high temperatures, roughly exceeding $150 \mathrm{~K}^{3}$ Therefore, strained InAs/GaInSb SL system appeared to be an excellent choice for the VLWIR detection. Unfortunately, the VLWIR InAs/GInSb material

${ }^{a)}$ Electronic mail: heather.haugan.ctr@us.af.mil. growth studies are still in immature stage of development. Many crystal growers tend to believe that the existence of an alloy layer can create a higher degree of disorder caused by alloy scattering, indium segregation, compositional interdiffusion, and unpredictable others. Therefore, controlling a precise gap and achieving high quality of materials using the ternary system can be more difficult than using the binary. So far, a majority of reported VLWIR photodiodes covering $50 \%$ cutoff wavelengths from 15 to $26 \mu \mathrm{m}$ at $10 \mathrm{~K}$ in the past years were based on minimally strained binary SL materials. ${ }^{4-7}$ Although there was a report of a ternary SL photodiode with a $50 \%$ cutoff wavelength of $21 \mu \mathrm{m}$ at $40 \mathrm{~K}$ using a $29 \mathrm{MLs}$ InAs/10 MLs $\mathrm{Ga}_{0.93} \mathrm{In}_{0.07} \mathrm{Sb}$ SLs with $1 \mathrm{ML}$ of InSb-like interfacial bonds, ${ }^{8}$ the authors added very little indium to their design and could not take advantages of alloying indium to the GaSb layer to improve Auger lifetimes. Therefore, their detectivity was about $3 \times 10^{9} \mathrm{~cm} \mathrm{~Hz}^{1 / 2} / \mathrm{W}$ at $40 \mathrm{~K}$.

In this Letter, we report ternary growth studies to develop this material for VLWIR detection. We select a SL structure of $47.0 \AA$ InAs/21.5 $\mathrm{Ga}_{0.75} \mathrm{In}_{0.25} \mathrm{Sb}$ proposed by Grein et al., ${ }^{2}$ theoretically adjusted for the greatest possible detectivity, and optimize a growth parameter for the best possible material quality. Since SL material quality is strongly related to the densities of nonradiative $\mathrm{SRH}$ recombination centers and the residual dopings, ${ }^{9}$ the impact of growth temperature $\left(\mathrm{T}_{\mathrm{g}}\right)$ on the photoresponse $(\mathrm{PR})$ intensity, and the charge carrier density and mobility will be investigated using photoconductivity and temperature-dependent Hall effect measurements. The $300 \mathrm{~K}$ recombination lifetime of a SL sample optimized for a strong PR signal will also be investigated by time-resolved pump-probe measurements.

The ternary SLs were grown by molecular beam epitaxy using elemental metals for Ga, In, and valved cracker cells for $\mathrm{As}$ and $\mathrm{Sb}$. The repeated SL stack and buffer layer were deposited on a lightly doped n-type GaSb (100) wafer, which provides a high enough isolation for electrical measurements. ${ }^{10}$ A series of $0.5 \mu \mathrm{m}$ thick $47.0 \AA$ InAs/21.5 $\mathrm{Ga}_{0.75} \mathrm{In}_{0.25} \mathrm{Sb}$ SLs were grown at $\mathrm{T}_{\mathrm{g}}$ between 390 and 
$470{ }^{\circ} \mathrm{C}$. To grow the intended sample structure, the growth rate combination of $1.20,0.33$, and $0.40 \AA / s$ was used for $\mathrm{GaSb}$, InAs, and InSb, respectively. The V/III flux ratio was set at minimum of 3 for both InAs and GaSb layers. Since monomeric $\mathrm{Sb}$ is the species critical to reducing densities of $\mathrm{SRH}$ recombination centers in the SL material, we set a cracking zone temperature at $950{ }^{\circ} \mathrm{C}$ for the series, which is close to the suggested value by the EPI Model $200 \mathrm{cc}$ Mark V Corrosive Series Valved Cracker. Interface between InAs and GaInSb layer was not intentionally controlled in order to compensate the residual strain in the SLs. Therefore, the strain of the SLs varied between $+0.2 \%$ and $0.0 \%$. Structural properties of grown samples such as SL period, residual strain, and overall layer thickness were assessed by highresolution transmission electron microscopy (HRTEM) and high-resolution $\mathrm{x}$-ray rocking curve (HRXRC) measurements. Figure 1(a) is a HRTEM image of a $47 \AA$ InAs $/ 21.5 \AA$ $\mathrm{In}_{0.25} \mathrm{Ga}_{0.75} \mathrm{Sb}$ SLs showing the first few SL periods adjacent to the substrate, wherein the amplitude contrast is optimized to delineate the individual layers in the structure. The average values for the individual layer thicknesses of InAs and GaInSb measured from this image were $45.7 \AA \pm 0.4 \AA$ and $22.8 \AA \pm 1.6 \AA$, which are close to their respective intended values of $47.0 \AA$ and $21.5 \AA$. In addition, quantitative strain analysis of these images was also performed using procedures described in a previous report. ${ }^{11}$ Figure $1(\mathrm{~b})$ shows the profile of the strain tensor $\varepsilon_{\mathrm{yy}}$ normal to the growth direction measured with respect to the GaSb substrate and averaged along the interface. Sharp negative spikes at the GaInSb-onInAs interface (denoted by arrows) are clearly observed, indicating that this interface is in tensile strain and that the dominant bond type at this interface is Ga-As. On the other hand, the regions corresponding to the InAs-on-GaInSb interface exhibits strain inversion, so that the overall strain at this interface is small. It is also observed that the GaInSb layers exhibit a strong compressive strain of approximately 0.03 , which is consistent with the high indium in this sample. Further analysis indicated that the net strain over several periods examined is to be about 0.0005 . With the measured period of $68.4 \AA$ strain balanced SL structure, we obtained the band gap of $45.3 \mathrm{meV}$ or a corresponding onset wavelength of $26.4 \mu \mathrm{m}$. The measured periods and strains of the growth temperature study series are listed in Table I.

Since the strength of PR signal is a strong function of the overall material quality, PR spectra were used to identify
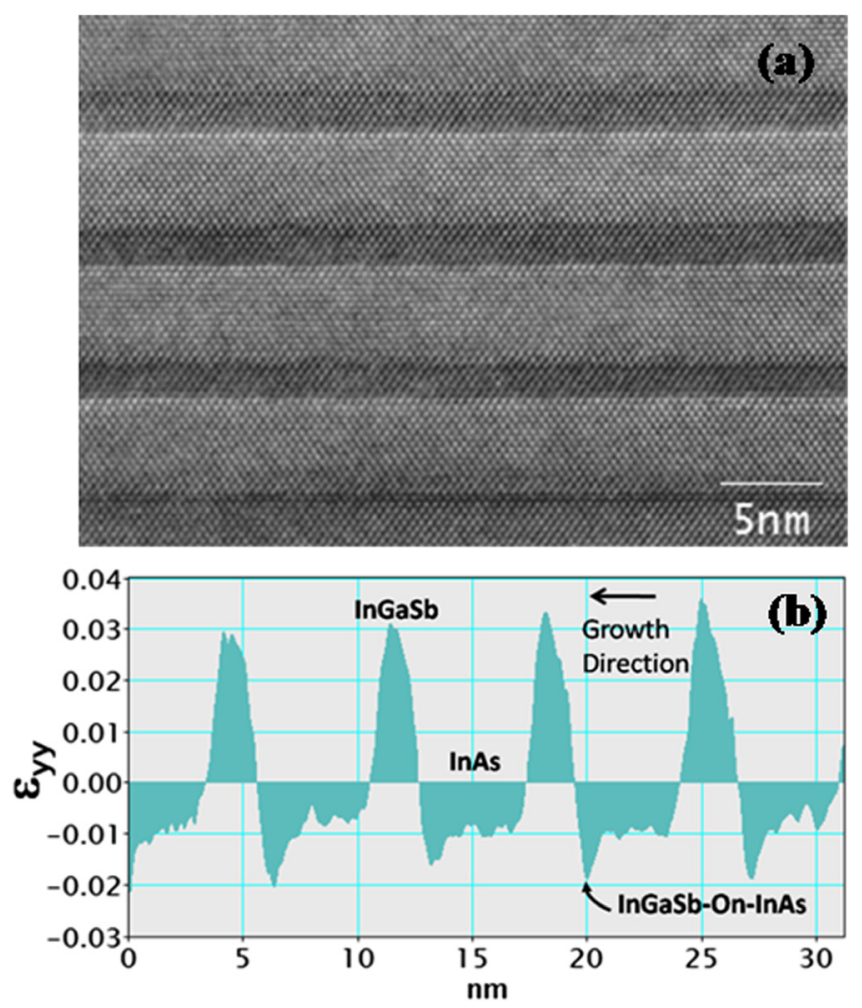

FIG. 1. (a) A HRTEM image of a $47 \AA$ InAs/21.5 $\operatorname{In}_{0.25} \mathrm{GaSb}_{0.75}$ superlattice showing the first few layers near the substrate. (b) The strain profile of the strain tensor $\varepsilon_{\mathrm{yy}}$ along the growth direction averaged parallel to the interface.

the spectral range of the material and strength of the optically excited signal. The spectra were collected with a Fourier transform infrared spectrometer at a temperature of $10 \mathrm{~K}$. Due to the relatively low resistivity of the samples, the photoconductivity was measured in the current biased mode, with a current of $0.5 \mathrm{~mA}$ between two parallel strips contact on the surface. Figure 2 shows the five PR spectra collected from the sample set with varying $\mathrm{T}_{\mathrm{g}}$ of 400, 410, 430, 440, and $450{ }^{\circ} \mathrm{C}$. Although the PR intensities are given in arbitrary units, the relative signal strengths can still be compared as the test conditions for all the samples were kept constant. We observe that the band gap energies of all SLs in this series are around $50 \pm 5 \mathrm{meV}$, while $\mathrm{PR}$ intensity gradually increases as $\mathrm{T}_{\mathrm{g}}$ increases from 400 to $440{ }^{\circ} \mathrm{C}$ by reaching a maximum at $440{ }^{\circ} \mathrm{C}$, and then drops by over an order of magnitude at higher temperatures. This result indicates that while

TABLE I. Summary of the measurements results for the sample set. The photoresponse and Hall results are from measurements at $10 \mathrm{~K}$. The cut-off wavelength $\lambda_{\mathrm{c}}$ is selected at the point where the intensity drops by $50 \%$. The PR intensity was measured at $100 \mathrm{meV}$ above the band gap. All of the samples were n-type.

\begin{tabular}{|c|c|c|c|c|c|c|c|c|}
\hline Sample & $\mathrm{T}_{\mathrm{g}}\left({ }^{\circ} \mathrm{C}\right)$ & $P(\AA)$ & $\varepsilon(\%)$ & $\mathrm{E}_{\mathrm{g}}(\mathrm{meV})$ & $\lambda_{\mathrm{c}}(\mu \mathrm{m})$ & PR intensity (arb. units) & Conc. $\left(\times 10^{11} \mathrm{~cm}^{-2}\right)$ & Mobility $\left(\mathrm{cm}^{2} / \mathrm{Vs}\right)$ \\
\hline SL1 & 390 & 67.8 & +0.18 & $\mathrm{X}$ & $\mathrm{X}$ & $\mathrm{X}$ & 9.1 & 6073 \\
\hline SL2 & 400 & 68.5 & +0.17 & 47.0 & 19.0 & 0.08 & 8.9 & 9887 \\
\hline SL3 & 410 & 67.5 & +0.17 & 43.8 & 20.1 & 0.32 & 7.1 & 11286 \\
\hline SL4 & 430 & 68.0 & +0.17 & 46.0 & 19.0 & 0.59 & 6.4 & 10502 \\
\hline SL5 & 440 & 67.5 & +0.16 & 53.0 & 17.0 & 1.09 & 5.2 & 9950 \\
\hline SL6 & 450 & 68.6 & +0.16 & 50.0 & 16.2 & 0.07 & 5.6 & 8529 \\
\hline SL7 & 460 & 67.7 & +0.11 & 60.0 & 15.7 & 0.06 & 280 & 112 \\
\hline SL8 & 470 & 67.5 & +0.00 & $\mathrm{X}$ & $\mathrm{X}$ & $\mathrm{X}$ & 1200 & 21 \\
\hline
\end{tabular}




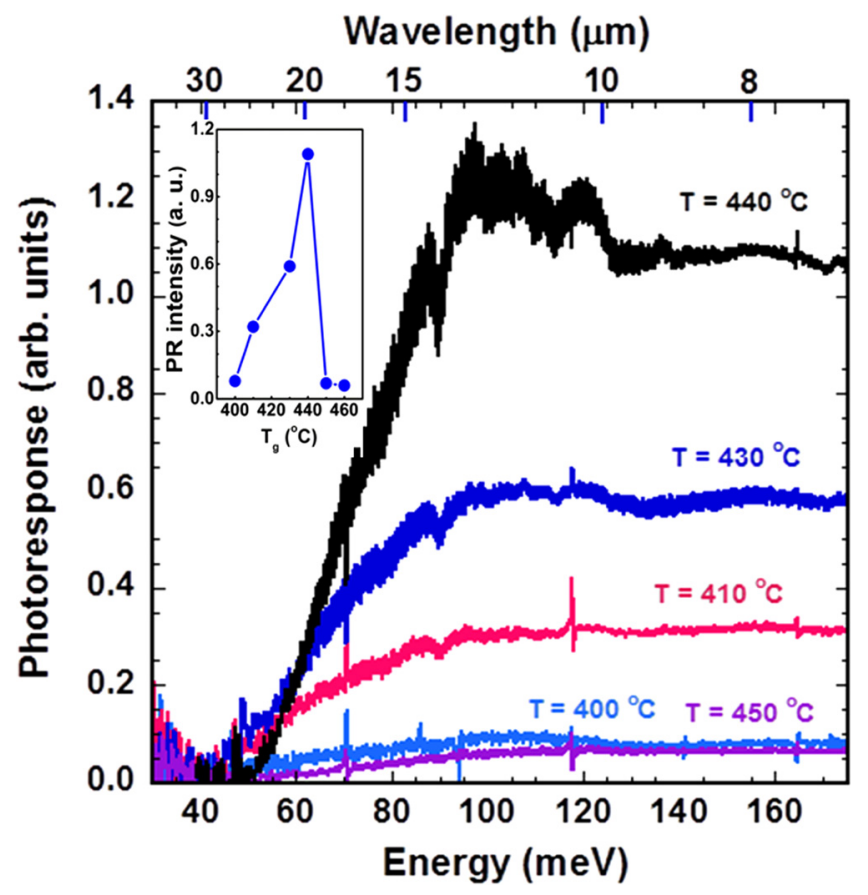

FIG. 2. Comparison of the photoresponse spectra of five $47.0 \AA$ InAs/21.5 $\mathrm{Ga}_{0.75} \mathrm{In}_{0.25} \mathrm{Sb}$ SL samples grown at $400-450^{\circ} \mathrm{C}$. The insert shows the PR intensity as a function of growth temperature $\left(\mathrm{T}_{\mathrm{g}}\right)$.

our MBE growth process used can reproduce a consistent gap near $50 \mathrm{meV}$ within a range of few meV. However, the PR intensity can be very sensitive to the minor variations of material quality. We observe a shallow growth window of $410-440{ }^{\circ} \mathrm{C}$ for given $\mathrm{V}$ fluxes and cracking zone temperatures we used. Outside this temperature window, the material quality deteriorates very rapidly.

To investigate transport properties, variable temperature resistivity and Hall effect measurements were performed on each sample in this set. The temperature dependence of the in-plane mobility for two SLs is shown in Figure 3(a), where the mobility is relatively temperature insensitive up to roughly $70 \mathrm{~K}$. The $10 \mathrm{~K}$ carrier density and mobility, along with the corresponding $\mathrm{T}_{\mathrm{g}}$, for each sample are listed in Table I. The impact of growth temperature on the carrier mobility measured at $10 \mathrm{~K}$ is summarized on Table $\mathrm{I}$ and illustrated in Figure 3(b). The mobility increases from 6000 to $11300 \mathrm{~cm}^{2} / \mathrm{Vs}$ as $\mathrm{T}_{\mathrm{g}}$ increases from 390 to $410^{\circ} \mathrm{C}$, gradually decreases to $8500 \mathrm{~cm}^{2} / \mathrm{Vs}$ as $\mathrm{T}_{\mathrm{g}}$ increases to $450{ }^{\circ} \mathrm{C}$, and then drops significantly for the two highest $\mathrm{T}_{\mathrm{g}}$ of 460 and $470{ }^{\circ} \mathrm{C}$. The background carrier density gradually decreases, reaching a minimum of $5.2 \times 10^{11} \mathrm{~cm}^{-2}$ at $440{ }^{\circ} \mathrm{C}$, with a corresponding mobility of roughly $10000 \mathrm{~cm}^{2} / \mathrm{Vs}$. Based on the transport data, transport properties rapidly deteriorate for $\mathrm{T}_{\mathrm{g}}$ above $450{ }^{\circ} \mathrm{C}$.

To measure the minority carrier lifetime, we performed time-resolved differential reflectivity measurements at $300 \mathrm{~K}$ on a SL sample. The pump was the output of an optical parametric amplifier (OPA), which was pumped by a regeneratively amplified Ti:Sapphire laser (CPA-2001, Clark-MXR, Inc.) with a repetition rate of $1 \mathrm{kHz}$. The wavelength of the OPA beam was tuned to $1.5 \mu \mathrm{m}$. The probe was the output of a continuous wave laser diode with a wavelength of $780 \mathrm{~nm}$. A silicon balanced detector with a bandwidth of $350 \mathrm{MHz}$

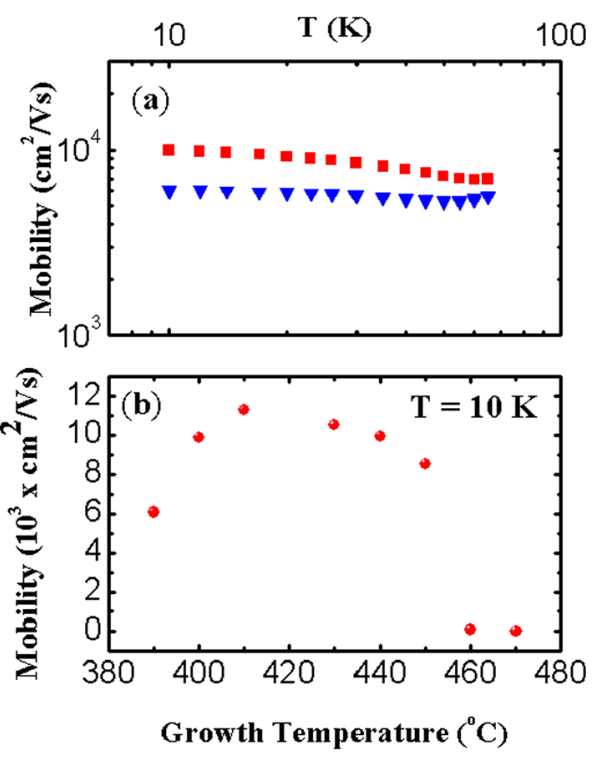

FIG. 3. (a) Temperature dependence of the carrier mobility as a function of $\mathrm{T}$ for SL samples grown at 440 (red solid squares) and $390^{\circ} \mathrm{C}$ (blue solid triangles). (b) The mobility as a function of growth temperature measured at $10 \mathrm{~K}$.

(PDB130A, Thorlabs, Inc.) was used to measure the difference in optical power between a reference arm of the probe beam and the probe beam reflected from the surface of the sample at the pump spot. By measuring the RF output of the balanced detector with a fast oscilloscope, we were able to measure the change in reflectivity due to the pump with a time resolution of $\sim 1 \mathrm{~ns}$. Figure 4 shows the result of this measurement at $300 \mathrm{~K}$. Here, we see multiple exponential decay components in the differential reflectivity, with the dominant contribution coming from an exponential with a decay time of $\sim 70 \mathrm{~ns}$. This should be interpreted as the lower limit for the actual recombination lifetime since the pumpprobe signal is likely to be affected by carrier diffusion both

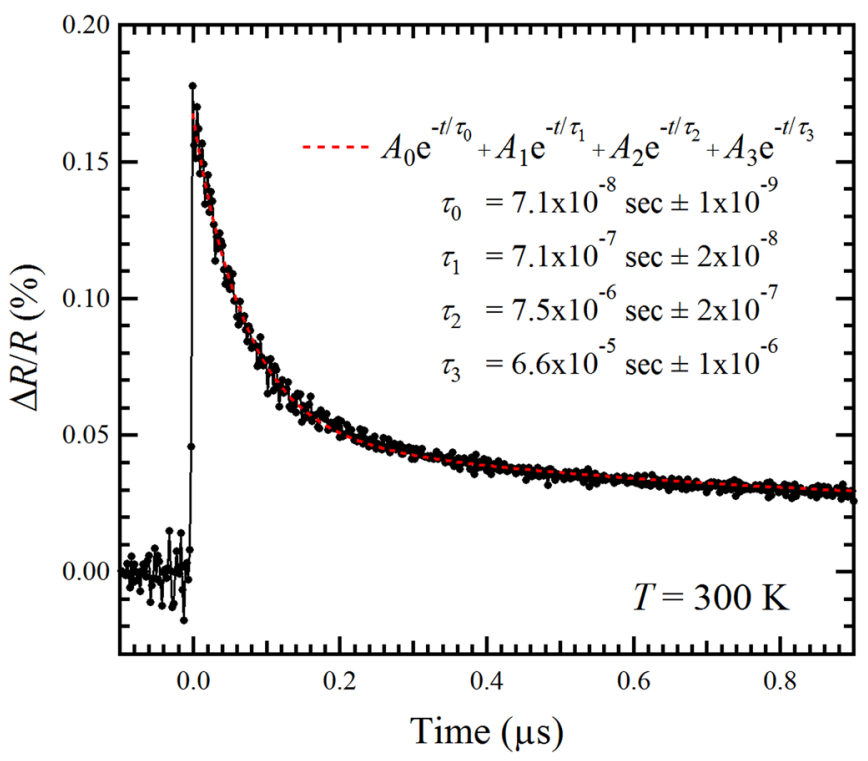

FIG. 4. Time-resolved differential reflectivity data for a $\sim 50 \mathrm{meV} 47 \AA$ InAs/ $21.5 \AA \mathrm{In}_{0.25} \mathrm{GaSb}_{0.75}$ superlattice sample at $300 \mathrm{~K}$. The data are fit with multiple exponetials, $A_{0} e^{-t / \tau_{0}}+A_{1} e^{-t / \tau_{1}}+A_{2} e^{-t / \tau_{2}}+A_{3} e^{-t / \tau_{3}}$, with the dominant exponential having a decay time of $\sim 70 \mathrm{~ns}$, where $A_{0}=1.2 \times 10^{-3} \pm 1 \times 10^{-4}$, $A_{1}=2.8 \times 10^{-4} \pm 1 \times 10^{-5}, A_{2}=1.3 \times 10^{-4} \pm 1 \times 10^{-5}$, and $A_{3}=1.1 \times 10^{-4}$ $\pm 1 \times 10^{-5}$. 
in the lateral and vertical directions, ${ }^{12}$ which contributes to a faster decay of the signal.

In conclusion, a combination of photoconductivity, temperature dependent Hall, and time-resolved differential reflectivity measurements was used to investigate the impact of $T_{g}$ on the material quality of VLWIR InAs/GaInSb SLs. For the studies, a series of $47.0 \AA \operatorname{InAs} / 21.5 \AA \mathrm{Ga}_{0.75} \mathrm{In}_{0.25} \mathrm{Sb}$ SLs were grown by MBE at $T_{g}$ ranging from 390 to $470{ }^{\circ} \mathrm{C}$. The results showed that our MBE growth process used to create a ternary SL structure produced a consistent band gap of $50 \pm 5 \mathrm{meV}$. However, the material quality of the grown samples assessed by PR intensity and Hall mobility was very sensitive to $T_{g}$. We observed a general trend of improving $\mathrm{PR}$ intensity as the temperature increases. The PR signal exhibited a maximum at $440{ }^{\circ} \mathrm{C}$, and then dropped rapidly to less than 0.1 a.u. at higher temperatures. The SLs were all residual n-type, but the mobility varied between 11300 and 21 $\mathrm{cm}^{2} / \mathrm{Vs}$. The mobility of the SL grown at $440^{\circ} \mathrm{C}$ was approximately $10000 \mathrm{~V} / \mathrm{cm}^{2}$ with a sheet carrier concentration of $5 \times 10^{11} \mathrm{~cm}^{-2}$, but the mobility precipitously dropped to 21 $\mathrm{cm}^{2} / \mathrm{Vs}$ at higher temperatures. The $300 \mathrm{~K}$ recombination lifetime of a SL sample that produced a strong PR signal was roughly $70 \mathrm{~ns}$.

The work of H. J. Haugan was performed under Air Force Contract No. FA8650-11-D-5801. The authors thank S. Fenstermaker and G. Landis for a technical assistance with the MBE system and sample preparation for the measurements, respectively. The lifetime data reported here were conducted by Rice University, and the research was sponsored by Air Force Research Laboratory under Agreement No. FA8650-07-5061.

${ }^{1}$ D. L. Smith and C. Mailhiot, J. Appl. Phys. 62, 2545 (1987).

${ }^{2}$ C. H. Grein, W. H. Lau, T. L. Harbert, and M. E. Flatté, Proc. SPIE 4795 , 39 (2002).

${ }^{3}$ C. H. Grein, J. Garland, and M. E. Flatté, J. Electron. Mater. 38, 1800 (2009).

${ }^{4}$ Y. Wei, A. Gin, M. Razeghi, and G. J. Brown, Appl. Phys. Lett. 80, 3262 (2002).

${ }^{5}$ H. Mohseni, Y. Wei, and M. Razeghi, Proc. SPIE 4288, 191 (2001).

${ }^{6}$ Y. Wei, A. Gin, M. Razeghi, and G. J. Brown, Appl. Phys. Lett. 81, 3675 (2002).

${ }^{7}$ H. Mohseni, M. Razeghi, G. J. Brown, and Y. S. Park, Appl. Phys. Lett. 78, 2107 (2001).

${ }^{8}$ E. H. Aifer, E. M. Jackson, G. Boishin, L. J. Whitman, I. Vurgaftman, J. R. Meyer, J. C. Culbertson, and B. R. Bennett, Appl. Phys. Lett. 82, 4411 (2003).

${ }^{9}$ H. J. Haugan, S. Elhamri, F. Szmulowicz, B. Ullich, G. J. Brown, and W. C. Mitchel, Appl. Phys. Lett. 92, 071102 (2008).

${ }^{10}$ W. C. Mitchel, S. Elhamri, H. J. Haugan, and G. J. Brown, "Electrical isolation of type II InAs/InGaSb supperlattices from GaSb substrates," J. Appl. Phys. (unpublished).

${ }^{11}$ K. Mahalingam, H. J. Haugan, G. J. Brown, K. G. Eyink, and B. Jiang, Proc. SPIE 8268, 826831 (2012).

${ }^{12}$ H. J. Haugan, G. J. Brown, S. Elhamri, S. Pacley, B. V. Olson, and T. F. Boggess, J. Appl. Phys. 111, 053113 (2012). 